Check for updates

Cite this: RSC Adv., 2018, 8, 29789

\title{
Different transition metal combinations of LDH systems and their organic modifications as UV protecting materials for polypropylene (PP)
}

\author{
Sajid Naseem, (D) *a Sunil P. Lonkar, ${ }^{\text {b }}$ Andreas Leuteritz ${ }^{a}$ \\ and Frederick J. W. J. Labuschagné (D) ${ }^{c}$
}

\begin{abstract}
In this research, the use of layered double hydroxides (LDHs) as ultraviolet (UV) light-protecting additives for PP is explored. Different LDHs, such as ZnTi, ZnSn, ZnGa, ZnCr and CdCr LDHs, were prepared and their UV absorptions were characterized. The ZnTi LDHs showed higher UV absorption than the other four metallic combinations and were further organically modified with dodecylbenzene sodium sulfonate (SDBS) and lauric acid (LA). Nanocomposites of polypropylene (PP) with four different types of LDHs, ZnTi, ZnSn, ZnTi-SDBS and ZnTi-LA, were prepared at concentrations of 5\%. The crystallinities and layered structures of all the metallic combinations of LDHs were characterized by wide angle X-ray spectroscopy (WAXS) and ultraviolet visible (UV-vis) absorption spectroscopy, and their crystal morphologies were studied by scanning electron microscopy (SEM). The decomposition and thermal properties of the nanocomposites and pure PP were analyzed by thermogravimetric analysis (TGA) and transmission electron microscopy (TEM) and by their photo-oxidation behavior. The addition of these organically modified and unmodified LDHs showed significant changes in the thermal decomposition of PP. The thermal stability of PP was increased to around $70{ }^{\circ} \mathrm{C}$ by the addition of SDBS-modified ZnTi LDHs (5\% by weight), and an increase in induction time of about $300 \%$ was determined.
\end{abstract}

Received 25th June 2018

Accepted 7th August 2018

DOI: $10.1039 / \mathrm{c} 8 \mathrm{ra05447a}$

rsc.li/rsc-advances

\section{Introduction}

Polypropylene (PP) has several advantages in daily life and is used worldwide for various applications. Many of its applications function in open environments; in many cases, PP is exposed to sunlight, which makes it susceptible to photodegradation. ${ }^{1}$ The chances of photodegradation of polypropylene (PP) from sunlight are very high; therefore, the stability and long life of PP mainly depend on ultraviolet light (UV) protection when it is used in sunlight. ${ }^{2-4}$ Sunlight can cause degradation and color loss of different polymers; consequently, the mechanical strength of the polymers decreases. Different types of polymers can be severely affected by aspects of the external environment, such as light, oxygen and temperature. Sunlight can produce reactive free radicals that can lead to a decrease in the strength of polymers. ${ }^{5,6}$

The basic strategy to avoid degradation of polymeric materials is to decrease the damage that occurs due to radiation and suppress radical formation. This can be accomplished using UV

${ }^{a}$ Leibniz-Institut für Polymerforschung Dresden e.V, Hohe Straße 6, Dresden, 01069, Germany.E-mail: naseem@ipfdd.de

${ }^{b}$ Department of Chemical Engineering, Khalifa University, Abu Dhabi, United Arab Emirates

'Department of Chemical Engineering, University of Pretoria, Lynwood Road, Pretoria, 0002, South Africa light absorbers, hydroxide decomposers and radical scavengers. Various types of additives are commercially available to protect polymers against UV degradation, including organic and inorganic additives. ${ }^{6}$ The common additives for UV absorbers in polymers are organic UV absorbers. Some issues arise when using only organic UV absorbers; for example, they can absorb energy from sunlight and dissipate it as heat, which can promote thermal degradation of the polymer matrix. Because they can leach out from the matrix, the efficiency of these UV absorbers is much lower. Also, there are other concerns, such as efficiency and, especially, long life efficiency; sometimes, these organic absorbers can leach out from the matrix. There are some environmental concerns in using only organic UV absorbers; for example, cinnamates, triazines and benzophenones are not environmentally friendly. These issues mostly occur when organic UV absorbers are used in high concentrations. ${ }^{6}$

The counterparts of organic UV absorbers are inorganic UV absorbers, which are currently in use. Inorganic UV absorbers are also useful, although they do have drawbacks. For example, $\mathrm{TiO}_{2}$ and $\mathrm{ZnO}$ are the most widely used inorganic UV absorbers because they are thermally stable compared to organic UV absorbers. Because inorganic UV absorbers are relatively large, polymer composites containing inorganic absorber particles are nontransparent, ${ }^{7,8}$ and these particles must be coated due to their ability to electrocatalytically degrade polymer 
structures. ${ }^{9-12}$ Inorganic fillers can also cause a severe decrease in the mechanical strengths of polymers if used alone., ${ }^{9,10}$ Although both organic and inorganic additives are used to protect polymers from different types of degradation, their use in polymers also has drawbacks.

To overcome the shortcomings of both types of additives, they must be combined to optimize their properties. If they can be combined together in a certain way, the benefits of both additives can be exploited and their shortcomings can be minimized. Using hybrid UV absorbers with organic anions hosted by inorganic cations in polymers has several advantages, such as increased thermal and photostability of the polymers. A mixture of inorganic and organic UV absorbers is advantageous over both organic and inorganic additives; the simplest examples are organic anions hosted by inorganic cations, such as layered double hydroxides (LDHs). LDHs are a special kind of anionic clay with a formula of $\left[\mathrm{M}_{1-x}{ }^{2+} \mathrm{M}_{x}{ }^{3+}(\mathrm{OH})_{2}\right]^{x+} \cdot\left[\left(\mathrm{A}^{n-}\right)_{x / n^{-}}\right.$ $\left.\cdot y \mathrm{H}_{2} \mathrm{O}\right]^{x-}$, where $\mathrm{M}^{2+}, \mathrm{M}^{3+}$ and $\mathrm{A}^{n-}$ are divalent metal cations, trivalent metal cations and interlayer anions, respectively. ${ }^{13}$ LDHs have a wide range of applications in polymers and elastomers; these materials have been widely studied for different applications in recent years. ${ }^{14}$ There are several possible methods to prepare different types of LDHs; for example, J. J. Bravo-Suárez et al. presented a summary of several possibilities to prepare LDHs. They studied almost the entire periodic table, theoretically proving that changing the metal ratio and changing the combination of metal species can change several properties. ${ }^{15}$

The interlayer anions of LDHs can also be replaced with different anions with different properties; thus, LDHs are unique materials with multifunctional applications. LDHs can act as host materials for different anions. There are several options for modification with organic and inorganic modifiers, and different functionalities can be created. These types of materials can be the best option to obtain desired properties for different types of polymers. These combinations of inorganic cations/organic anions (host-guest) can function as optimized additives for polymers. ${ }^{16}$ There are numerous possible replacement anions with different functionalities. Due to this replacement with different types of anions, LDHs can be used as multifunctional materials for polymers, resulting in the fabrication of polymers as multifunctional composites. ${ }^{17-21}$

One less frequently studied application of LDHs for polymers is their UV absorbing characteristics. Few researchers have studied the UV absorption of LDHs with different viewpoints in the last few years. Moreover, the UV absorption and protection capabilities of LDHs and the modification of LDHs for UV protection of polymers is even less frequently discussed..$^{22-26}$ The main aim in protecting PP against UV is to add LDHs with good UV light absorbing capability. If the LDHs have good ability to absorb most UV light, it is highly possible to decrease or completely prevent the photo-degradation of PP. LDH structures may allow intercalation of organic UV absorbers, thus enlarging the processing window for compounding. Generally, organomodification of LDHs is important to achieve highly dispersed particles on the nanometer scale without sacrificing the optical properties of the compound in the visible light region. ${ }^{15}$

Our group has worked in the field of LDHs for many years and has produced different types of LDHs for multifunctional applications in polymers and elastomers..$^{16,27,28}$ In this research, different types of LDH systems were prepared with different types of metal salts; then, their UV absorbing qualities were verified by UV-vis spectroscopy. After that, selected LDHs were modified with two organic modifiers, dodecylbenzene sodium sulfonate (SDBS) and lauric acid (LA), which have good UV lightabsorbing capabilities. Then, these organically modified LDHs and unmodified LDHs were melt-compounded in PP, and the photo-degradation and thermal degradation of all the PP composites were compared.

\section{Experimental techniques and characterization}

\section{Materials}

The chemicals used for the synthesis and modification of different LDHs were $\mathrm{Zn}\left(\mathrm{NO}_{3}\right)_{2} \cdot 6 \mathrm{H}_{2} \mathrm{O}, \mathrm{TiCl}_{4}$, urea, $\mathrm{Na}_{2} \mathrm{Co}_{3}$, $\mathrm{Ga}\left(\mathrm{NO}_{3}\right)_{2} \cdot 4 \mathrm{H}_{2} \mathrm{O}, \mathrm{Cr}\left(\mathrm{NO}_{3}\right)_{2} \cdot 9 \mathrm{H}_{2} \mathrm{O}, \mathrm{NaOH}, \mathrm{SnCl}_{4}, \mathrm{Cd}\left(\mathrm{NO}_{3}\right)_{2^{-}}$ $\cdot 4 \mathrm{H}_{2} \mathrm{O}, \mathrm{Cr}\left(\mathrm{NO}_{3}\right)_{2} \cdot 9 \mathrm{H}_{2} \mathrm{O}$, polypropylene (PP) (Borealis MFI 2.53.5/10 min, $M_{\mathrm{w}}: 310000$, density: 0.9), PP-g-MA (Exxon MobilExxelor, MA content: $0.5 \%$ to $1 \%$, density: $0.91 \mathrm{~g} \mathrm{~cm}^{-3}$, MFI110), lauric acid (LA) and dodecylbenzene sodium sulfonate (SDBS). All these chemicals were purchased from SigmaAldrich, were of analytical grade and were used without further purification.

\section{Synthesis of LDHs}

The ZnTi and ZnSn LDHs were synthesized by a urea hydrolysis method. In a typical procedure, a $3: 1$ molar ratio of $\mathrm{Zn}\left(\mathrm{NO}_{3}\right)_{2} \cdot 6 \mathrm{H}_{2} \mathrm{O}$ and $\mathrm{TiCl}_{4}$ was used. $0.22 \mathrm{ml}$ of $\mathrm{TiCl}_{4}, 1.19 \mathrm{~g}$ of $\mathrm{Zn}\left(\mathrm{NO}_{3}\right)_{2} \cdot 6 \mathrm{H}_{2} \mathrm{O}$ and $3 \mathrm{~g}$ of urea were dissolved in $100 \mathrm{ml}$ of deionized water under vigorous stirring. The reactants were aged for 48 hours at $130{ }^{\circ} \mathrm{C}$. A $4: 1$ molar ratio of $\mathrm{Zn}\left(\mathrm{NO}_{3}\right)_{2}$ $\cdot 6 \mathrm{H}_{2} \mathrm{O}$ and $\mathrm{SnCl}_{4}$ was used. $0.04 \mathrm{M}$ aqueous solution of $\mathrm{Zn}\left(\mathrm{NO}_{3}\right)_{2} \cdot 6 \mathrm{H}_{2} \mathrm{O}, 0.01 \mathrm{M} \mathrm{SnCl}_{4}$ aqueous solution and $0.16 \mathrm{M}$ urea solution were mixed together and heated at $100{ }^{\circ} \mathrm{C}$ for 30 hours. ${ }^{24}$

The $\mathrm{ZnGa}, \mathrm{ZnCr}$ and $\mathrm{CdCr}$ LDHs were prepared by a coprecipitation method. A solution of $\mathrm{Zn}\left(\mathrm{NO}_{3}\right)_{2} \cdot 6 \mathrm{H}_{2} \mathrm{O}$ and $\mathrm{Ga}\left(\mathrm{NO}_{3}\right)_{2} \cdot 4 \mathrm{H}_{2} \mathrm{O}$ in a $2: 1$ molar ratio was added dropwise to $1 \mathrm{M}$ $\mathrm{Na}_{2} \mathrm{CO}_{3}$ solution. The $\mathrm{pH}$ was maintained at 8 by the addition of sodium hydroxide solution. The aging time of the precipitates was 22 hours at $80{ }^{\circ} \mathrm{C} .{ }^{29} \mathrm{Zn}\left(\mathrm{NO}_{3}\right)_{2} \cdot 6 \mathrm{H}_{2} \mathrm{O}$ and $\mathrm{Cr}\left(\mathrm{NO}_{3}\right)_{2} \cdot 9 \mathrm{H}_{2} \mathrm{O}$ solutions in a $2: 1$ molar ratio were mixed dropwise with sodium hydroxide and $\mathrm{Na}_{2} \mathrm{CO}_{3}$; the $\mathrm{pH}$ was maintained at 9 . The aging time of the precipitates was 24 hours at $60{ }^{\circ} \mathrm{C}^{30}$ Solutions of $\mathrm{Cd}\left(\mathrm{NO}_{3}\right)_{2} \cdot 4 \mathrm{H}_{2} \mathrm{O}$ and $\mathrm{Cr}\left(\mathrm{NO}_{3}\right)_{2} \cdot 9 \mathrm{H}_{2} \mathrm{O}$ in a $2: 1$ molar ratio were mixed dropwise with sodium hydroxide, and the $\mathrm{pH}$ was maintained at 8 . The aging time of the precipitates was 18 hours at $100{ }^{\circ} \mathrm{C} .{ }^{31}$ All five different types of LDHs were filtered and washed. The LDHs were dried in an oven at $60{ }^{\circ} \mathrm{C}$ overnight. 


\section{Modification of LDHs}

In a typical method, $4 \mathrm{~g}$ of SDBS was dissolved in $100 \mathrm{ml}$ of deionized water. $1 \mathrm{~g}$ of $\mathrm{ZnTi} \mathrm{LDH}$ was mixed in SDBS solution and stirred for 24 hours at $80^{\circ} \mathrm{C}$ under nitrogen. The modified ZnTi LDHs were filtered, washed and dried in an oven at $60{ }^{\circ} \mathrm{C}$ overnight. ${ }^{32}$ A mixture of LA : ZnTi LDH in a ratio of $4: 1$ at $120^{\circ} \mathrm{C}$ was melted in a strictly inert atmosphere for 3 hours and then thoroughly washed with warm $\mathrm{MeOH}$.

\section{Polypropylene nanocomposite preparation}

The nanocomposites were prepared by a melt intercalation method using a twin screw extruder microcompounder (5 $\mathrm{cm}^{3}$ ). The first master batch with a ratio of LDHs and PP-g-MA of $1: 3$ was prepared using a DSM twin screw microcompounder $\left(5 \mathrm{~cm}^{3}\right)$. A processing temperature of $180{ }^{\circ} \mathrm{C}$ was used with a screw speed of $200 \mathrm{rpm}$, and the residence time was $06 \mathrm{~min}$. In the next stage, the master batch was diluted in PP; a processing temperature of $180{ }^{\circ} \mathrm{C}$ was used with a screw speed of $200 \mathrm{rpm}$ for $05 \mathrm{~min}$. In the final composite, the composition was PP : PP-g-MA : LDH (80: 15 : 5), i.e. $5 \% \mathrm{LDH}$ loading. ${ }^{16,19}$ The abbreviations and compositions are given in Table 1.

\section{Characterization techniques}

Powder X-ray diffractogram patterns were recorded using a 2circle diffractometer (XRD 3003 h/h, Seifert-FPM Freiberg/Sa., now: GE Sensing \& Inspection Technologies $\mathrm{GmbH}$ ) by wide angle X-ray spectroscopy (WAXS). The scanning was performed in the range of $5^{\circ}$ to $35^{\circ}$. Thermogravimetric analysis (TGA) was performed using a TGA Q5000 instrument from TA Instruments under air between $40^{\circ} \mathrm{C}$ and $600{ }^{\circ} \mathrm{C}$ with a heating rate of $10^{\circ} \mathrm{C} \mathrm{min}^{-1}$. The surfaces of the samples were observed by scanning electron microscopy (SEM); the images were taken with an Ultra Plus (Fa. Zeiss), and transmission electron microscopy (TEM) images were taken with a Libra $200 \mathrm{MS}$ (Fa. Zeiss). Samples were prepared for SEM by distributing the LDHs on a stub and sputter-coating it with $3 \mathrm{~nm}$ platinum. UVvis spectroscopy was carried out using a Lambda 800 instrument from Perkin Elmer. Infrared spectra (FTIR) were recorded with a Nicolet 5SX-FTIR Spectrometer with OMNIC software. The spectra were obtained in the absorbance range of $4000 \mathrm{~cm}^{-1}$ to $400 \mathrm{~cm}^{-1}$. Photo-oxidation studies were performed using the method described in our previous article, in which a SEPAP 12.24 instrument equipped with mercury lamps was used. ${ }^{16}$

Table 1 Details of the samples and their abbreviations

\begin{tabular}{llll}
\hline Sample & LDH (wt\%) & PP (wt\%) & PP-g-MA (wt\%) \\
\hline SPL-0 & - & 84 & 16 \\
SPL-1 & ZnTi (5\%) & 80 & 15 \\
SPL-2 & ZnSn (5\%) & 80 & 15 \\
SPL-3 & ZnTi-SDBS (5\%) & 80 & 15 \\
SPL-4 & ZnTi-LA (5\%) & 80 & 15
\end{tabular}

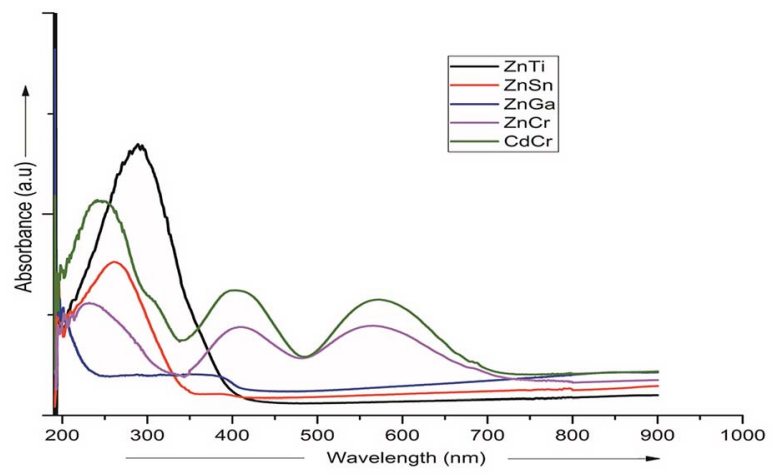

Fig. 1 UV-vis spectra of the different types of LDHs.

\section{Results and discussion}

\section{UV-vis spectroscopy, XRD and SEM analysis of modified and unmodified LDHs}

UV-vis absorption spectroscopy was used to analyze the various nanoparticles. Fig. 1 shows the results of UV-vis spectroscopy for five different types of LDHs. From the UV-vis spectroscopy results, it is evident that the LDHs with different metal combinations have different UV-vis absorptions. In Fig. 1, it can be seen that the ZnTi and ZnSn LDHs show a strong single UV-vis absorption peak in the UV-vis range of the graph, in contrast to the other LDHs. There is no strong UV-vis absorption peak in the case of $\mathrm{ZnGa}$, as can be seen in Fig. 1. Meanwhile, $\mathrm{ZnCr}$ and CdCr show three UV-vis absorption peaks, one in the UV range and two in the visible range, as shown in Fig. 1. Based on the evidence of the UV-vis absorption peaks, ZnTi was chosen for further organic modification due to its strongest UV-vis light absorption. The UV-vis absorption peak in the ZnTi LDHs is similar to those of $\mathrm{ZnO}$ and $\mathrm{TiO}_{2}$, which have been used previously for UV protection of different polymers. ${ }^{6,33,34}$

WAXS was used to characterize the structures and crystallinities of the different LDHs. SEM analysis was used to determine the surface morphologies and crystal structures of the different LDHs. Further investigation of these five types of LDHs can be seen in the WAXS graphs and SEM images in Fig. 2. The $\mathrm{XRD}$ graph represents the typical crystal reflections and layered structures of the LDHs. From the XRD graphs in Fig. 2, the layered structures of ZnTi and ZnSn can be confirmed. Layered structures of the other three types of LDHs cannot be confirmed through their XRD curves. The layered structures of ZnTi and $\mathrm{ZnSn}$ can also be seen in the SEM images in Fig. 2. The SEM images of the other three types of LDHs show fluffy structures. There is no clear indication in the XRD and SEM images that $\mathrm{ZnGa}$, ZnCd or $\mathrm{CdCr}$ are layered structures. ${ }^{19}$ On the basis of these results, it can be stated that ZnTi and ZnSn show better layered structures and UV absorption compared to the other three LDH combinations. Scheme 1 represents the process by which ZnTi LDHs can absorb UV light and can protect PP. ${ }^{\mathbf{1 6}}$

Organomodification is important to achieve highly dispersed particles in PP. In Fig. 3, typical reflections of crystals can be observed in the XRD graphs at (003) and (006). The peaks 

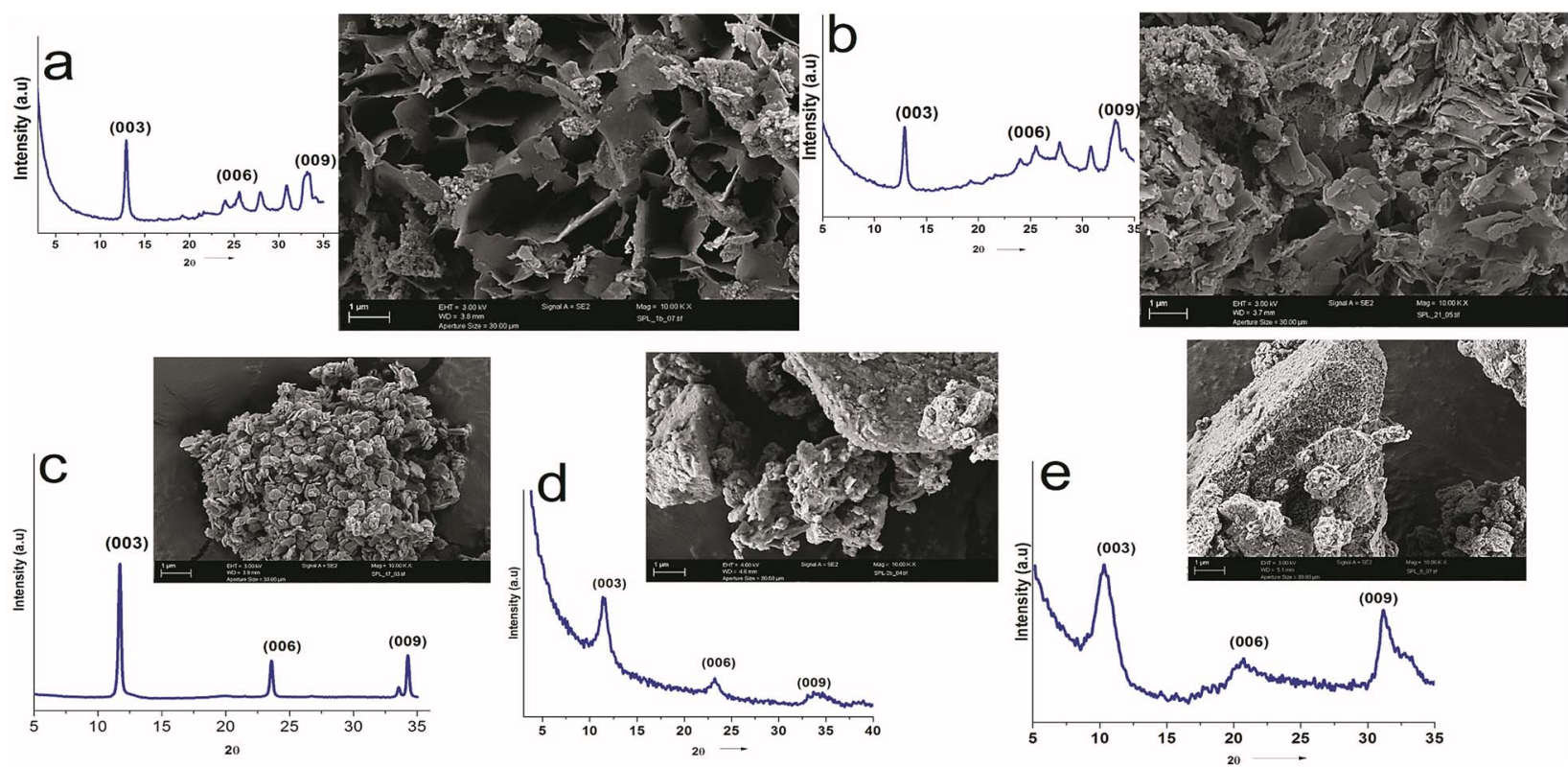

Fig. 2 XRD and SEM results of the (a) ZnTi (b) ZnSn (c) ZnGa (d) $\mathrm{ZnCr}$ and (e) $\mathrm{CdCr}$ LDHs.

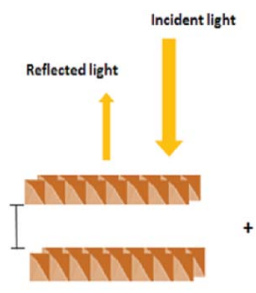

ZnTi LDH

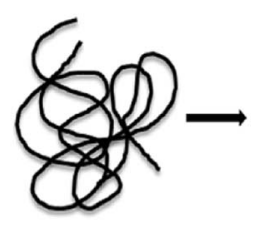

PP+PP-g-MA

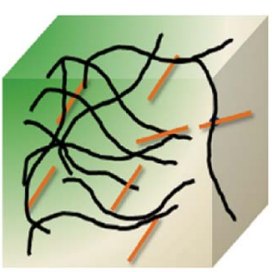

Scheme 1 Schematic of the UV absorption effect of ZnTi in PP. ${ }^{16}$

indicate the good crystallinity of the LDHs. The reflection peak at (003) indicates the repeating unit of the LDH; it is the sum of one metal hydroxide layer and the spacing between two adjacent layers. This peak appears at a $2 \theta$ angle of about $12^{\circ}$ in the
ZnTi LDH; it moves to about $3^{\circ}$ in the case of ZnTi-SDBS and $2.5^{\circ}$ in the case of ZnTi-LA. This indicates the intercalation of ZnTi LDH and enhancement of the interlayer distances of the ZnTi LDH layers. ${ }^{19}$ Schematic representations of the ZnTi LDH before and after modification with LA and SDBS are also shown in Fig. $3 .^{16}$ The interlayer spacing is enhanced due to SDBS and LA, as confirmed by the XRD results shown in Fig. $3 .{ }^{16}$ Organomodification of ZnTi was achieved successfully with SDBS (sodium dodecyl benzene sulphonate) and LA (lauric acid).

\section{Structural characterization of PP nanocomposites}

The most common crystalline forms of PP are the monoclinic $\alpha$ and hexagonal $\beta$ forms. The $\alpha$ crystalline form is the most common in PP, while the $\beta$ crystalline form is found only in special cases of quenching or when using different types of nucleating agents. ${ }^{35}$ Fig. 4 represents the XRD results of the PP and PP/LDHs. It can be observed in Fig. 4 that there is an

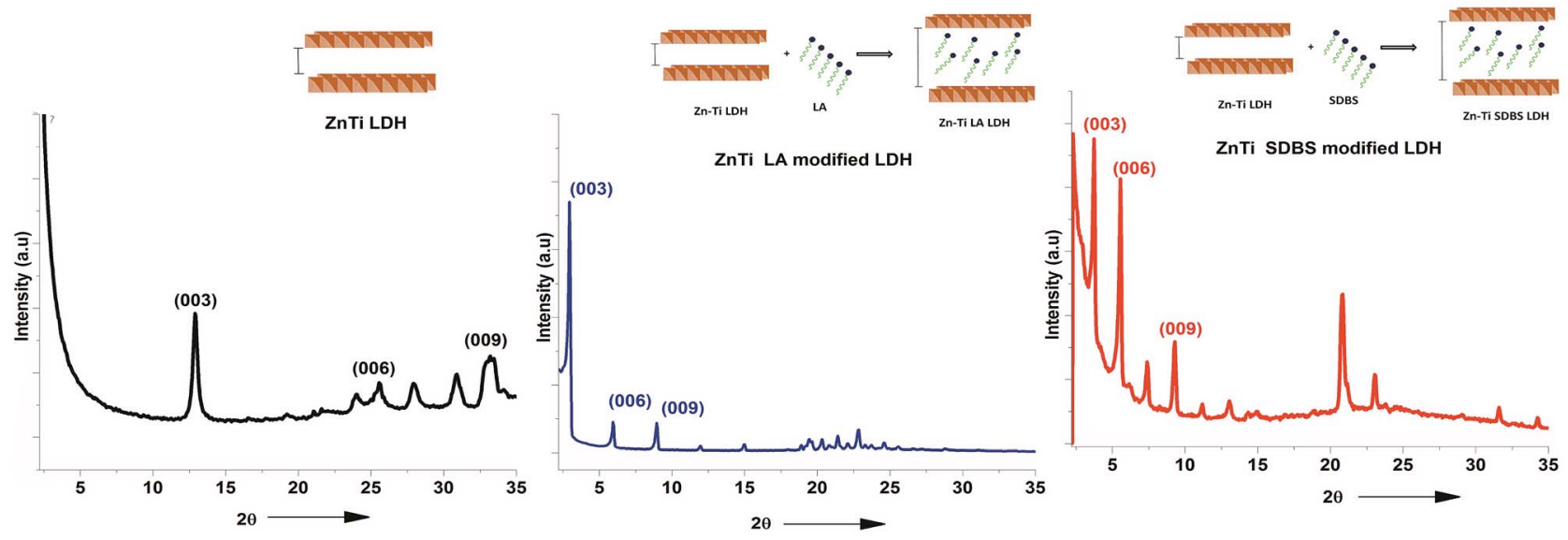

Fig. 3 XRD results of ZnTi LDH, ZnTi LA and ZnTi SDBS and schematics showing the intercalation of organic molecules. 


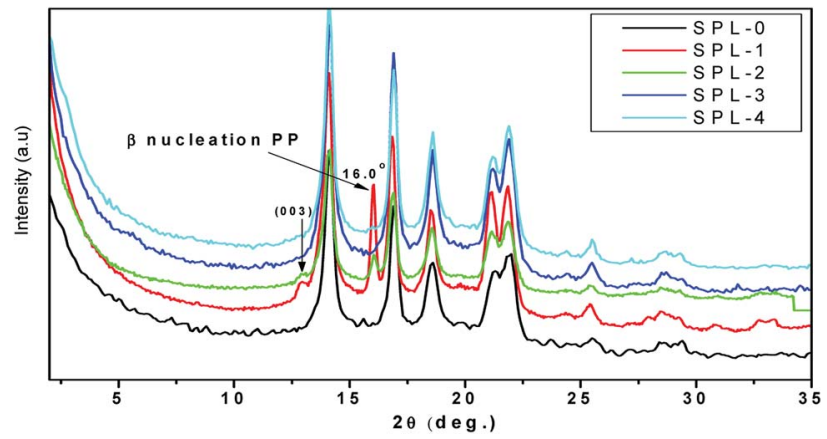

Fig. 4 XRD results showing the beta nucleation of PP.

intercalation effect of the LDHs in PP. More $\beta$ nucleation occurs in $\mathrm{PP} / \mathrm{ZnTi}$ and $\mathrm{PP} / \mathrm{ZnSn}$, while there is no $\beta$ phase in $\mathrm{PP} / \mathrm{ZnTi}$ SDBS or PP/ZnTi-LA; this shows the good dispersion of organically modified LDHs in PP. ${ }^{36-38} \mathrm{ZnTi}$ and $\mathrm{ZnSn}$ acted as a nucleating agent and developed a $\beta$ phase. ${ }^{35}$ It was previously reported that other inorganic nanoparticles can act as $\beta$ nucleating agents in the PP matrix, such as $\mathrm{CaCo}_{3}, \mathrm{Al}_{2} \mathrm{O}_{3}$ and $\mathrm{ZnO}^{35}$ In Fig. 4 , peaks of $\beta$ nucleation can be observed at $2 \theta=16^{\circ}$ in the cases of PP/ZnTi and PP/ZnSn. The surface morphologies of all the PP/LDHs were determined by TEM analysis, as shown in Fig. 5. From the TEM images, it can be seen that the organically modified LDHs are well dispersed in PP. From the TEM images, it can be seen that the intercalated nanocomposites were synthesized; tactoids are present in all the composites, as shown in Fig. 5. Intercalated tactoids can be observed in PP/ ZnTi-SDBS and PP/ZnSn-LA in Fig. 5. In PP/ZnTi-SDBS and PP/ ZnSn-LA, more dispersion can be seen in Fig. 5. The XRD and TEM evidence show better intercalation for the organomodified PP/LDHs over PP/ZnTi and PP/ZnSn.

\section{Thermal properties}

Polymers are subject to physical and mechanical degradation with increasing temperature. The thermal degradation behavior of these nanocomposites and neat PP were studied using TGA, and the data are shown graphically in Fig. 6(a and b). In TGA analysis, the mass of a sample is monitored as a function of temperature or as a function of time while the sample is maintained at a controlled temperature. The LDH-filled PP nanocomposites showed enhanced thermal stability, as can be seen in Fig. 6(a and b). The thermal stability increased more significantly in the case of the ZnTi-SDBS LDHs, by almost $70{ }^{\circ} \mathrm{C}$. Usually, the thermal stability increases marginally with the addition of clay to $\mathrm{PP} \cdot{ }^{39} \mathrm{The}$ onset temperature of the degradation of PP increased by $22{ }^{\circ} \mathrm{C}$ over that of pure PP by the addition of $3 \mathrm{wt} \%$ clay. ${ }^{40}$ Previously, $\mathrm{TiO}_{2}$ was used in different ratios in PP; the onset of degradation was found to improve compared to that of neat PP, which is $29.88^{\circ} \mathrm{C}$, by the addition of $1.5 \mathrm{wt} \% \mathrm{TiO}_{2} \cdot{ }^{41}$ Fig. 6 shows that the presence of LDHs causes distinct changes in the thermal decomposition behavior of PP in comparison to unfilled PP. Fig. 6(a and b) show the TGA graphs and derivative TGA graphs, respectively (Table 2).

The thermal stability increased by around $70^{\circ} \mathrm{C}$ by the addition of $5 \%$ ZnTi-SDBS LDHs, as can be seen in Fig. 6(b). The thermal stability of polymers can be understood in terms of two temperatures: (T0.1), at which 10\% weight loss occurs, and (T0.50), at which 50\% weight loss occurs. Fig. 6(c) shows a comparison of these two temperatures of pure PP and the LDHs/PP composites. The onset of decomposition is delayed in the case of the ZnTiSDBS/PP composite. ${ }^{42}$ It is clear from Fig. $6(\mathrm{a}-\mathrm{c})$ that the dispersion and organomodification of LDHs play significant roles in the thermal stability of the PP composites. ${ }^{43}$ This enhancement of the thermal stability of PP may be due to hindrance of the thermal motion of the PP chains due to the presence of LDHs. ${ }^{41}$
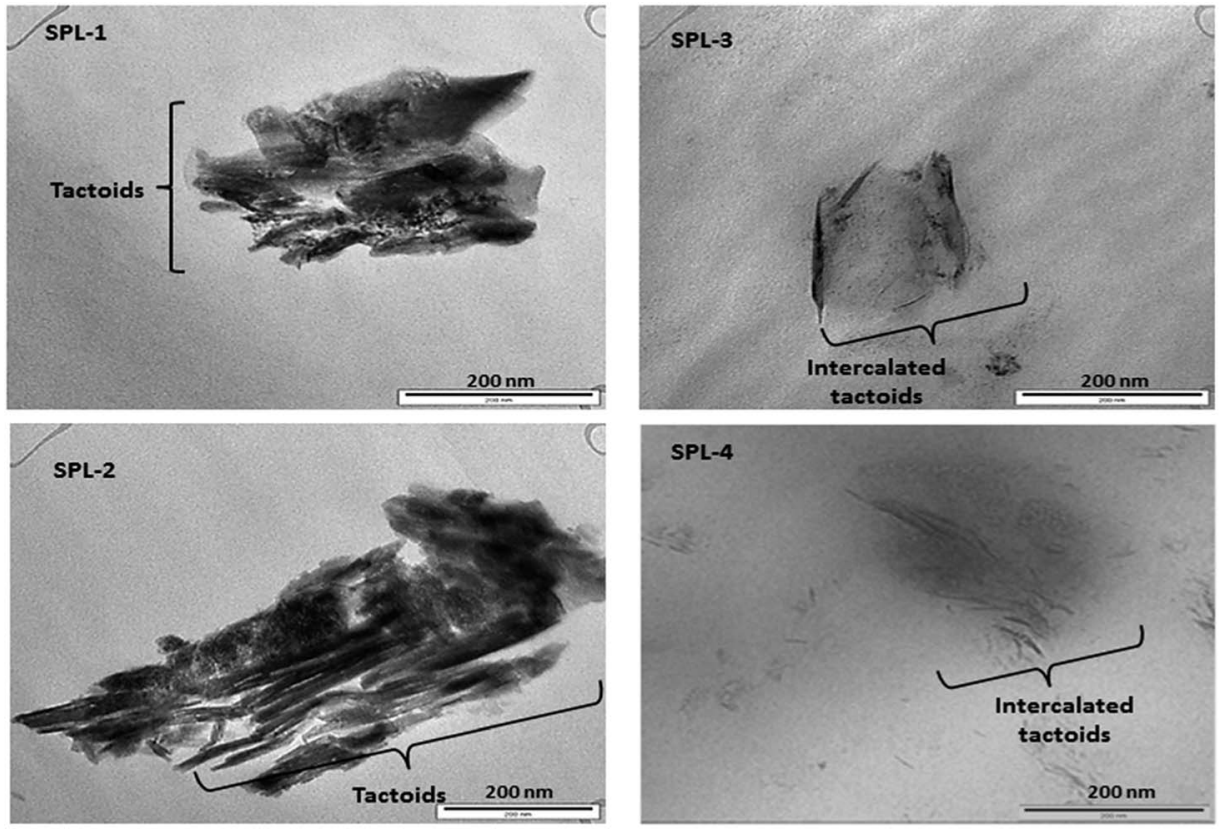

Fig. 5 TEM results showing the tactoids. 

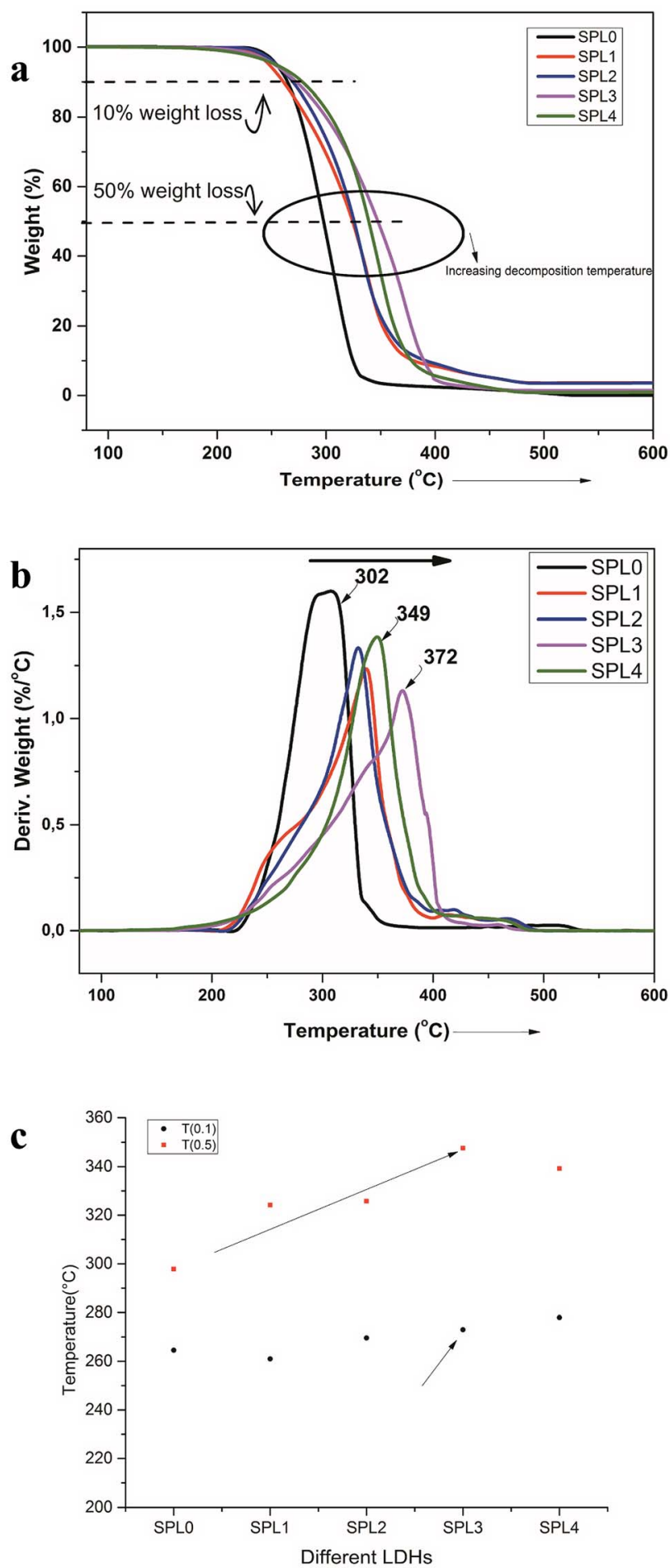

Fig. 6 (a) TGA and (b) derivative TGA of PP and the PP + LDHs composites. (c) Influence of different LDHs on the decomposition temperature of PP at $10 \%$ weight loss $\mathrm{T}(0.1)$ and $50 \%$ weight loss $\mathrm{T}(0.5)$.

\section{Photo-oxidation studies}

Fig. 7 presents FTIR spectra showing the degradation occurring in the PP nanocomposites. In Fig. 7, it is shown that degradation of PP occurs in all the samples on exposure to UV light. The graph shows different times ( 0 to $70 \mathrm{~h}$ ) of exposure to UV light. Carbonylated products and hydroxylated products are
Table 2 Temperature at which maximum degradation occurs in different LDH/PP composites

\begin{tabular}{ll}
\hline LDHs & $\begin{array}{l}T_{\max } \text { at } \\
\text { which maximum degradation occurs }\left({ }^{\circ} \mathrm{C}\right)\end{array}$ \\
\hline SPL0 & 302 \\
SPL1 & 339 \\
SPL2 & 332 \\
SPL3 & 372 \\
SPL4 & 349
\end{tabular}

representative of the degradation of $\mathrm{PP}$, as shown in Fig. 7. The traces of carbonyl groups for $0 \mathrm{~h}$-exposed $\mathrm{PP}$ are probably due to thermal oxidation during processing. The major products of degradation that can be observed in Fig. 7 are carbonylated (1700 to $1800 \mathrm{~cm}^{-1}$ ) and hydroxylated $\left(3300-3600 \mathrm{~cm}^{-1}\right)$ products. The carbonylated and hydroxylated products increased as the time of exposure increased, as can be seen in Fig. $7 .^{44}$ This photo-oxidation study was performed on all the samples of PP, and the combined results are shown in Fig. 8(a). The carbonylated and hydroxylated products were further used to characterize the photo-oxidation kinetics of all the nanocomposites. In Fig. 8(a), it can be seen that ZnTi-SDBS showed the highest stability effects on PP against photo-oxidation. All the other LDHs showed relatively less stability toward photo-oxidation in PP compared to the ZnTi-SDBS LDHs. A schematic of the addition of ZiTi-SDBS to PP is shown in Scheme 2. In Scheme 2, ZnTi-SDBS absorbs more light compared to the ZnTi LDH in Scheme 1; this may be due to the enhanced interlayer distance and the presence of SDBS in the layers of ZnTi LDHs. Not only the interlayer distance but the modifiers, functional groups and sizes of the modifiers are important. Also, the dispersion of sulphonate modifiers is better than that of other modifiers. As the size of modifiers increases, the intercalation of polymer chains within the interlayer regions of the LDHs in the nanocomposites is facilitated. ${ }^{45}$ These phenomena can also be seen in the UV-vis reflectance spectra shown in Fig. 8(b). The addition of ZnTi-SDBS to PP showed a good UV stabilization effect compared to other types of LDHs, as can be seen in Fig. 8(a and $\mathrm{b})$.

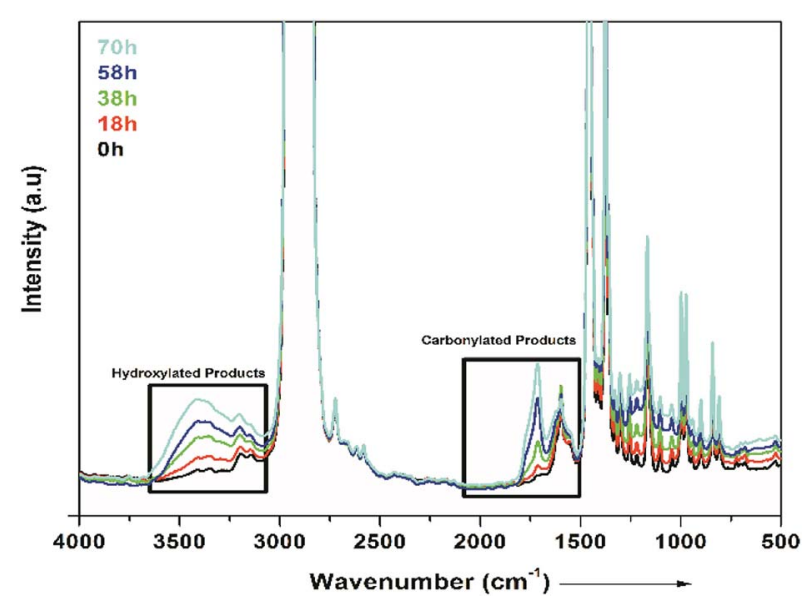

Fig. 7 FTIR spectra of the PP/ZnTi LDHs composites. 

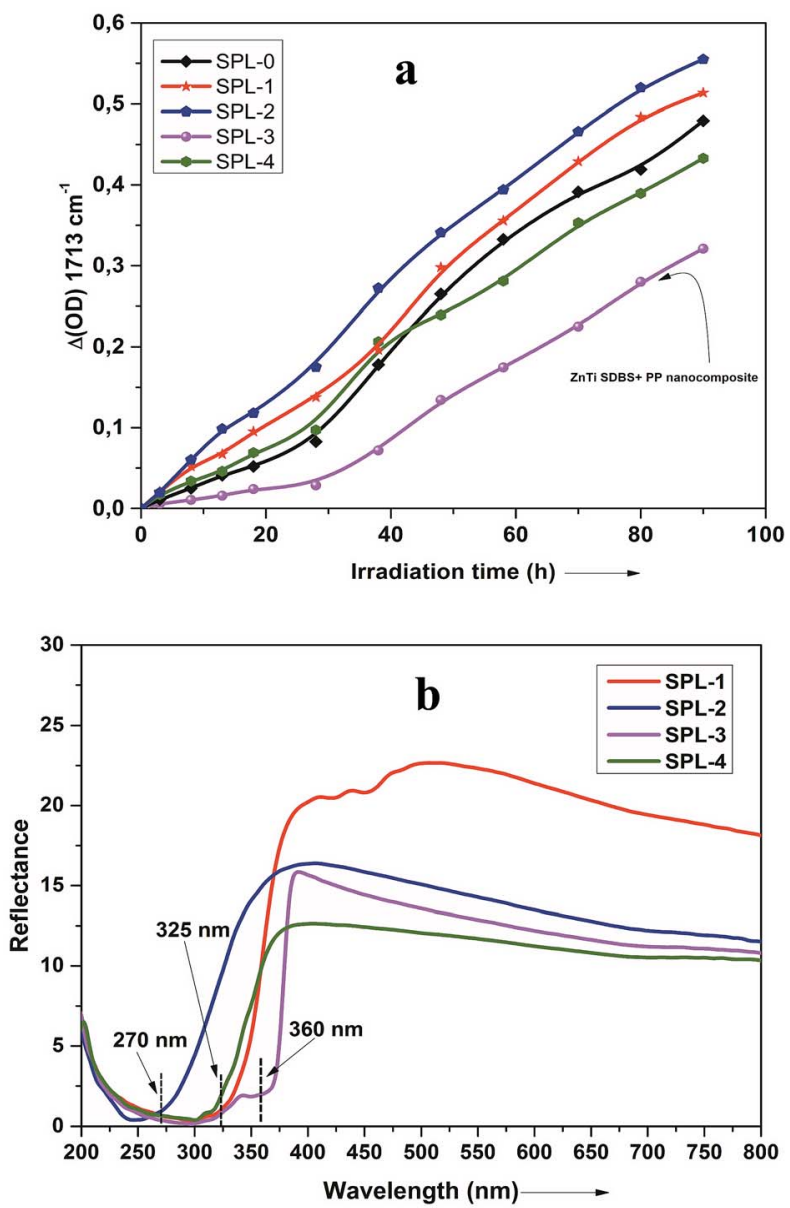

Fig. 8 (a) Photo-oxidation kinetics and (b) UV-vis reflectance spectra of the PP + LDH composites.

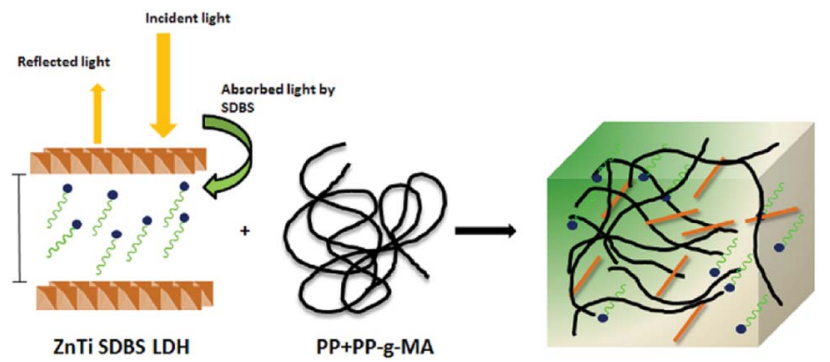

Scheme 2 Schematic of the effects of ZnTi SDBS on the UV absorption of PP. ${ }^{16}$

\section{Conclusion}

UV-vis-absorbing LDHs were prepared and modified with organic modifiers. The ZnTi LDH modified with SDBS showed the highest UV absorbing effect among the LDHs. The protecting capability of PP against photo and thermal degradation was enhanced by the addition of ZnTi-SDBS LDHs, which is important and can be used in other polymers. The degradation temperature of PP was enhanced by $70{ }^{\circ} \mathrm{C}$ by the addition of only 5\% of ZnTi-SDBS LDHs; increasing the LDHs amount may further enhance these properties. Also, this enhancement is greater than that of previously described inorganic additives and clays. This research can provide a new method for protecting polymers from UV-vis degradation by using different types of LDHs compared to conventional use of organic and inorganic UV-vis protecting agents. Depending on the application of the final product, different types of LDHs with combinations of organic anions and metallic cations can be synthesized. In the case of UV-vis absorbing LDHs, these results appear to open a new field of research in UV-vis absorbing LDHs for polymers; this may be used in different applications with a combination of polymers, such as in solar cells, electrostatic devices, LED devices and other energy-related applications.

\section{Conflicts of interest}

There are no conflicts to declare.

\section{Acknowledgements}

The authors are thankful to the Leibniz Institute of Polymer Research, Dresden-Germany for providing research facilities. The authors are also thankful to Dr R. P. Singh, NCL-Pune INDIA, for providing the facilities for the photo-oxidation kinetics studies.

\section{References}

1 Y. Gao, Y. Zhao, L. Qiu, Z. Guo, D. O'Hare and Q. Wang, Polym. Compos., 2017, 36, 1937-1947.

2 Y. Feng, D. Li, Y. Wang, D. G. Evans and X. Duan, Polym. Degrad. Stab., 2006, 91, 789-794.

3 H. Chai, Y. Lin, D. G. Evans and D. Li, Ind. Eng. Chem. Res., 2008, 47, 2855-2860.

4 R. Charifou, F. Gouanvé, R. Fulchiron and E. Espuche, J. Polym. Sci., Part B: Polym. Phys., 2015, 53, 782-794.

5 G. Wang, S. Xu, C. Xia, D. Yan, Y. Lin and M. Wei, RSC Adv., 2015, 5, 23708-23714.

6 Y. Peng, W. Wang, J. Cao and Y. Huang, J. Appl. Polym. Sci., 2017, 134, 44597.

7 D. Sengupta, P. Das, B. Mondal and K. Mukherjee, Renewable Sustainable Energy Rev., 2016, 60, 356-376.

8 T. J. Turton and J. R. White, Polym. Degrad. Stab., 2001, 74, 559-568.

9 B. K. Deka and T. K. Maji, Composites, Part A, 2011, 42, 21172125.

10 W. Shi, Y. Lin, S. Zhang, R. Tian, R. Liang, M. Wei, D. G. Evans and X. Duan, Phys. Chem. Chem. Phys., 2013, 15, 18217-18222.

11 P.-O. Bussière, J. Peyroux, G. Chadeyron and S. Therias, Polym. Degrad. Stab., 2013, 98, 2411-2418.

12 V. P. Gordienko and Y. A. Dmitriev, Polym. Degrad. Stab., 1996, 53, 79-87.

13 E. N. Kalali, X. Wang and D.-Y. Wang, J. Mater. Chem. A, 2016, 4, 2147-2157.

14 D. Basu, A. Das, K. W. Stöckelhuber, U. Wagenknecht and G. Heinrich, Prog. Polym. Sci., 2014, 39, 594-626. 
15 J. J. Bravo-Suárez, E. A. Páez-Mozo and S. T. Oyama, Quim. Nova, 2004, 27, 601-614.

16 S. P. Lonkar, A. Leuteritz and G. Heinrich, RSC Adv., 2013, 3, 1495-1501.

17 Q. Wang, J. Wu, Y. Gao, Z. Zhang, J. Wang, X. Zhang, X. Yan, A. Umar, Z. Guo and D. O'Hare, RSC Adv., 2013, 3, 2601726024.

18 A. Leuteritz, B. Kutlu, J. Meinl, D. Wang, A. Das, U. Wagenknecht, et al., Mol. Cryst. Liq. Cryst., 2012, 556, 107-113.

19 B. Kutlu, A. Leuteritz, L. Häußler, U. Oertel and G. Heinrich, Polym. Degrad. Stab., 2014, 102, 9-14.

20 B. Nagendra, A. Das, A. Leuteritz and E. B. Gowd, Polym. Int., 2016, 65, 299-307.

21 D. Yan, Y. Zhao, M. Wei, R. Liang, J. Lu, D. G. Evans and X. Duan, RSC Adv., 2013, 3, 4303-4310.

22 B. Schwenzer, J. R. Neilson, K. Sivula, C. Woo, J. M. J. Fréchet and D. E. Morse, Thin Solid Films, 2009, 517, 5722-5727.

23 B. Schwenzer, K. M. Roth, J. R. Gomm, M. Murr and D. E. Morse, J. Mater. Chem., 2006, 16, 401-407.

24 M. Shao, J. Han, M. Wei, D. G. Evans and X. Duan, Chem. Eng. J., 2011, 168, 519-524.

25 S. B. Tyagi, A. Kharkwal, Nitu, M. Kharkwal and R. Sharma, Solid State Sci., 2017, 63, 93-102.

26 Y. Peng, W. Wang, J. Cao and X. Guo, RSC Adv., 2015, 5, 41230-41237.

27 D. Basu, A. Das, D.-Y. Wang, J. J. George, K. W. Stockelhuber, R. Boldt, A. Leuteritz and G. Heinrich, $R S C A d v$., 2016, 6, 26425-26436.

28 A. Das, D.-Y. Wang, A. Leuteritz, K. Subramaniam, H. C. Greenwell, U. Wagenknecht and G. Heinrich, J. Mater. Chem., 2011, 21, 7194-7200.

29 G. S. Thomas and P. V. Kamath, Solid State Sci., 2006, 8, 1181-1186.
30 C. G. Silva, Y. Bouizi, V. Fornés and H. García, J. Am. Chem. Soc., 2009, 131, 13833-13839.

31 Y. Guo, H. Zhang, L. Zhao, G.-D. Li, J.-S. Chen and L. Xu, J. Solid State Chem., 2005, 178, 1830-1836.

32 J. He, M. Wei, B. Li, Y. Kang, D. G. Evans and X. Duan, Preparation of Layered Double Hydroxides, in Layered Double Hydroxides, ed. X. Duan and D. G. Evans, Springer Berlin Heidelberg, Berlin, Heidelberg, 2006, pp. 89-119.

33 J. Jiménez Reinosa, P. Leret, C. M. Álvarez-Docio, A. del Campo and J. F. Fernández, Bol. Soc. Esp. Ceram. Vidrio, 2016, 55, 55-62.

34 T. W. Quadri, L. O. Olasunkanmi, O. E. Fayemi, M. M. Solomon and E. E. Ebenso, ACS Omega, 2017, 2, 8421-8437.

35 H. Zhao and R. K. Y. Li, Polymer, 2006, 47, 3207-3217.

36 J. Cao, Y. Zheng and T. Lin, Polym. Test., 2016, 55, 318-327.

37 M. M. Favaro, M. C. Branciforti and R. E. S. Bretas, Mater. Res., 2009, 12, 455-464.

38 J. Kotek, I. Kelnar, J. Baldrian and M. Raab, Eur. Polym. J., 2004, 40, 679-684.

39 H. Sangeeta, B. Neelima and J. P. Jog, Polym. Eng. Sci., 2002, 42, 1800-1807.

40 N. S. Jafari, A. S. Javad, A. Hossein and M. Ahmad, J. Appl. Sci., 2007, 7, 2480-2484.

41 S. K. Esthappan, S. K. Kuttappan and R. Joseph, Polym. Degrad. Stab., 2012, 97, 615-620.

42 F. R. Costa, U. Wagenknecht and G. Heinrich, Polym. Degrad. Stab., 2007, 92, 1813-1823.

43 B. Nagendra, C. V. S. Rosely, A. Leuteritz, U. Reuter and E. B. Gowd, ACS Omega, 2017, 2, 20-31.

44 A. P. Gupta, U. K. Saroop and V. Gupta, J. Appl. Polym. Sci., 2007, 106, 917-925.

45 F. R. Costa, A. Leuteritz, J. Meinl, U. Wagenknecht and G. Heinrich, Macromol. Symp., 2011, 301, 46-54. 